Case Report

\title{
Gastric Smooth Muscle Hamartomas Mimicking Polyps in a Dog: A Case Description and a Review of the Literature
}

\author{
Marian A. Taulescu, ${ }^{1}$ Irina Amorim, ${ }^{2}$ Fatima Gärtner, ${ }^{2}$ Laura Fãrcaş, ${ }^{1}$ \\ Mircea V. Mircean, ${ }^{3}$ and Cornel Cătoi ${ }^{1}$ \\ ${ }^{1}$ Pathology Department, Faculty of Veterinary Medicine, University of Agricultural Sciences and Veterinary Medicine, \\ 3-5 Calea Mãnãștur Street, 400372 Cluj-Napoca, Romania \\ ${ }^{2}$ Department of Pathology and Molecular Immunology of the Institute of Biomedical Sciences Abel Salazar (ICBAS), \\ University of Porto, Rua Jorge Viterbo Ferreira Nr. 228, 4050-313 Porto, Portugal \\ ${ }^{3}$ Department of Internal Medicine, Faculty of Veterinary Medicine, University of Agricultural Sciences and Veterinary Medicine, \\ 3-5 Calea Mãnãştur Street, 400372 Cluj-Napoca, Romania
}

Correspondence should be addressed to Marian A. Taulescu; taulescumarian@yahoo.com

Received 25 June 2013; Accepted 27 August 2013

Academic Editors: N. D. Giadinis, S. Hecht, and R. L. Santos

Copyright (C) 2013 Marian A. Taulescu et al. This is an open access article distributed under the Creative Commons Attribution License, which permits unrestricted use, distribution, and reproduction in any medium, provided the original work is properly cited.

\begin{abstract}
This report presents a case of two smooth muscle hamartomas of the stomach in a 10-year-old male Boxer. The clinical history of the animal was of chronic vomiting, weight loss, and intermittent gastric distension, and it died because of chronic and congestive heart failure. Gross, histology, and immunohistochemistry (IHC) exams were performed. On necropsy, in the pyloric region of the stomach, two closely related polypoid growths between 10 and $15 \mathrm{~mm}$ in diameter were identified. On the cut sections, both polyps presented white to gray color, with homogenous architecture and well-defined limits. The thickness of the submucosal layer was seen to be increased to $1 \mathrm{~cm}$. No other gastric alterations were identified by the necropsy exam. Histologically, both masses growth consisted of hyperplastic glands lined by foveolar epithelium, arranged in a papillary or branching pattern, and supported by a core of well-vascularised and marked smooth muscle tissue interspersed between glands. No dysplastic cells and mitotic figures were observed in these lesions. Immunohistochemistry revealed a strong cytoplasm labelling for smooth muscle actin of the bundles around the mucosal glands. To our knowledge, this is the first report of smooth muscle hamartomas mimicking multiple gastric polyps in dogs.
\end{abstract}

\section{Introduction}

Gastric polyps (GP) are sessile or pedunculated growths that arise from the mucosa and protrude into the gastric lumen as the result of either hyperplasia or neoplasia [1]. GP occur sporadic in dogs [2,3], cats [4], cattle [5], and horses [6]; they are observed during gastric endoscopy or necropsy. GP are often in the pyloric region, and the affected animals usually do not show any clinical signs however. GP can produce vomiting after food intake, weight loss, and bleeding when these lesions reach a considerable size and rarely cause pyloric stenosis [2,3]. Macroscopically, GP are described as solitary or multiple bulging sessile or pedunculated formations, with an irregular and arborescent surface [7]. In human pathology, various types of nonneoplastic gastric polyps, including hyperplastic, inflammatory fibroid, xanthoma, hamartomatous of the Peutz-Jeghers type, juvenile, gastric polyps in Cowden disease, and gastric polyps in Cronkhite-Canada syndrome, are described [8]. In domestic animals, there is a lack of data about the histological features of the gastric polyps. Based on histological findings, two gastric polyps have been distinguished: hyperplastic and inflammatory (benign lymphoid) [1]. The pathogenesis of development of the GP in dogs is still unknown, but previous reports suggest a possible hereditary predisposition in French Bulldog $[3,9]$.

\section{Case Description}

This short communication describes an unprecedented case of gastric hamartomatous polyps in a 10 -year-old male Boxer. 


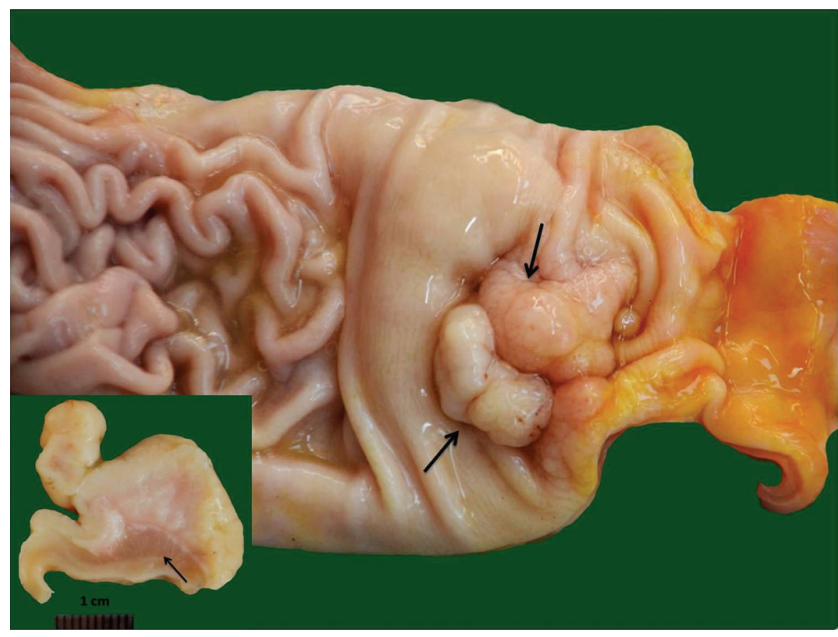

FIgURE 1: Photograph of the opened stomach showing two intraluminal and closely related polyp-like masses, measuring approximately $15 \mathrm{~mm}$ in diameter that was easily seen in the pyloric region (arrows). The inset illustrates the morphological aspects of the crosssection of the polypoid mass with a white to gray and dense structure and muscular layer thickening (arrow).

The animal was presented for examination with history of chronic vomiting, weight loss, and intermittent gastric distension. No endoscopy or other paraclinical investigations were made. The animal died 1 month later, due to congestive stage of heart chronic failure. During the necropsy, gastric lesions were collected, fixed in $10 \%$ buffered formalin, and submitted to histological examination. Tissues samples were routinely processed, dehydrated, and embedded in paraffin wax. Four $\mu \mathrm{m}$ consecutive sections were cut and stained with haematoxylin and eosin. Immunohistochemistry was also performed using the avidin-biotin-peroxidase complex (ABC) method, employing the monoclonal antisera muscular $\alpha$-actin (clone HHF35, Dako). Negative controls for each sample were prepared by replacing the primary antibody with mouse IgG1 Negative Control (Code X0931, Dako, Denmark). Semiquantitative assessment of immunohistochemical intensity was performed and scored negative $(-)$, weak $(+)$, moderate $(++)$, and intense $(+++)$.

On necropsy, luminal fluid and gases accumulation and two closely related polypoid growths were identified in the pyloric region of the stomach: one pedunculated measuring $1.5 \mathrm{~cm}$ in diameter and another sessile and dense on palpation with $1.0 \mathrm{~cm}$ in diameter. On the cut sections, both polyps present white to gray color, with homogenous architecture and well-defined limits; thickening of the muscular layer was also macroscopically observed (Figure 1). No other gastric alterations were identified; however, the necropsy exam also revealed bilateral ventricular dilation, severe and chronic pulmonary edema and diffuse pulmonary anthracosis, chronic and diffuse liver congestion, cystic mucinous hyperplasia of the gallbladder mucosa, and multifocal nodular exocrine pancreatic hyperplasia.

Histologically, both gastric masses projected above the level of the surrounding mucosa into the gastric lumen. The

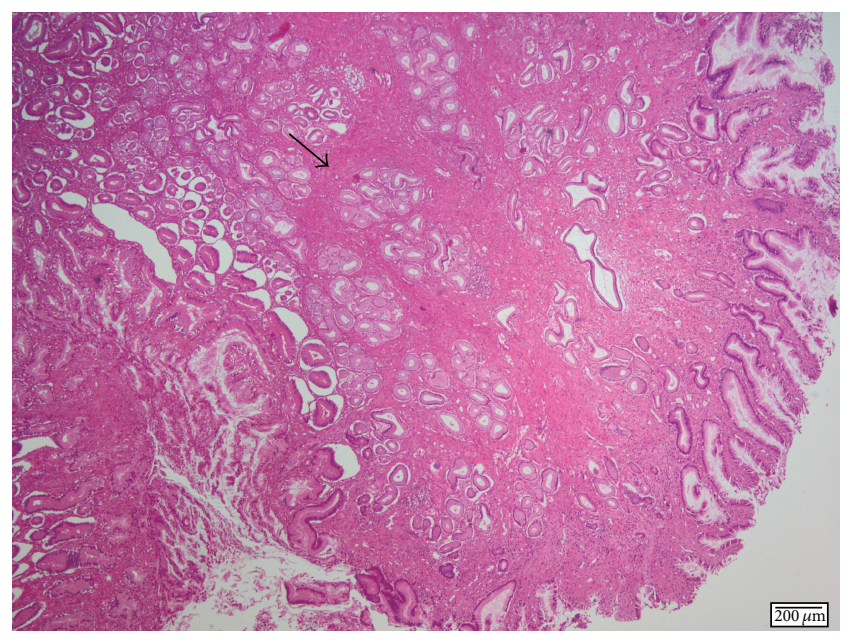

FIGURE 2: The histological exam of a section of the polypoid mass showing a branching framework consisting of numerous bands of smooth muscle cells (arrow) with intervening glandular tissue and covered by a mild to moderate foveolar hyperplasia. HE stain. Bar = $200 \mu \mathrm{m}$.

lesions were covered by hyperplastic foveolar epithelium with minimal branching and cystic dilatation, supported by an extensive smooth-muscle proliferation, with an elongated, arborized pattern of polyp formation (Figure 2). The deep area of the gastric polyp revealed several bands of smooth muscle cells between the gastric antral-type glands lined by well-differentiated mucinous epithelium and mild mononuclear cells infiltration consisting of lymphocytes, plasma cells, and hystiocytes (Figure 3). Neither cytological atypia nor mitotic figures were observed in this lesion. The presence of muscle fibres in the lamina propria was further confirmed by immunohistochemistry which revealed a strong cytoplasm labelling for smooth muscle actin of the bundles around the mucosal glands (Figure 4). No histological changes were found in the adjacent mucosa of the polypoid growths. The histopathological findings along with the immunohistochemical results suggested a diagnosis of canine gastric hamartomatous polyp.

\section{Discussion}

Hamartoma is a tumor-like malformation composed of an abnormal mixture of normal tissue elements or an abnormal proportion of a single element [10]. By definition, hamartomas are congenital lesions, with limited propensity for growth, and normally paralleling the growth of the host; however, they may not be detected until later in life [10]. In dogs, hamartomas are more commonly found in the skin [11], but some vascular hamartomas of the cerebrum [12] and lung [13] are also documented.

The gastric hamartomatous polyp is predominantly composed by hyperplastic mucosal glands, smooth muscle bundles in the mucosal layer, and asymptomatic lesions found incidentally in fewer than $0,1 \%$ of all endoscopy examinations [14]. 


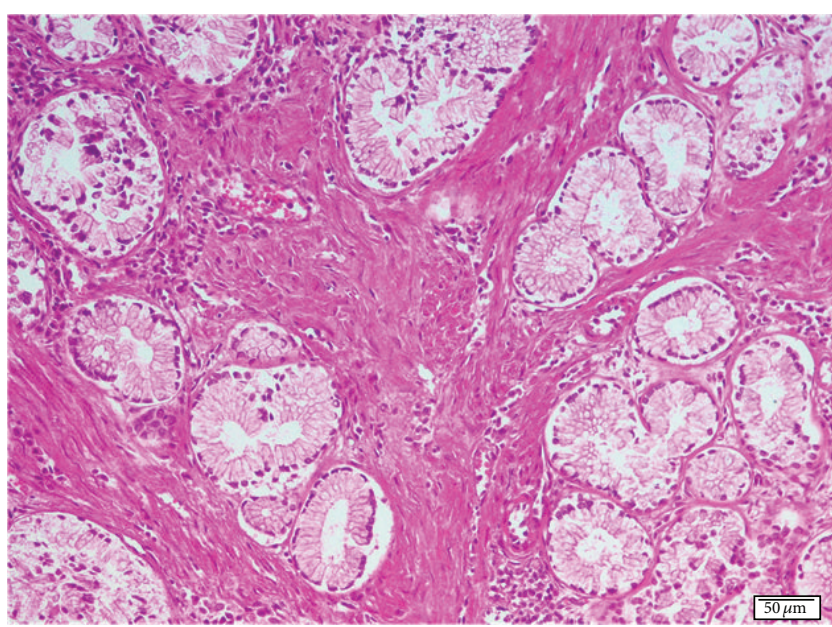

FIGURE 3: High power photomicrograph of a section of the polypoid mass showing several bands of well-differentiated smooth muscle cells between gastric antral-type glands lined by tall columnar mucinous epithelium. HE stain. Bar $=50 \mu \mathrm{m}$.

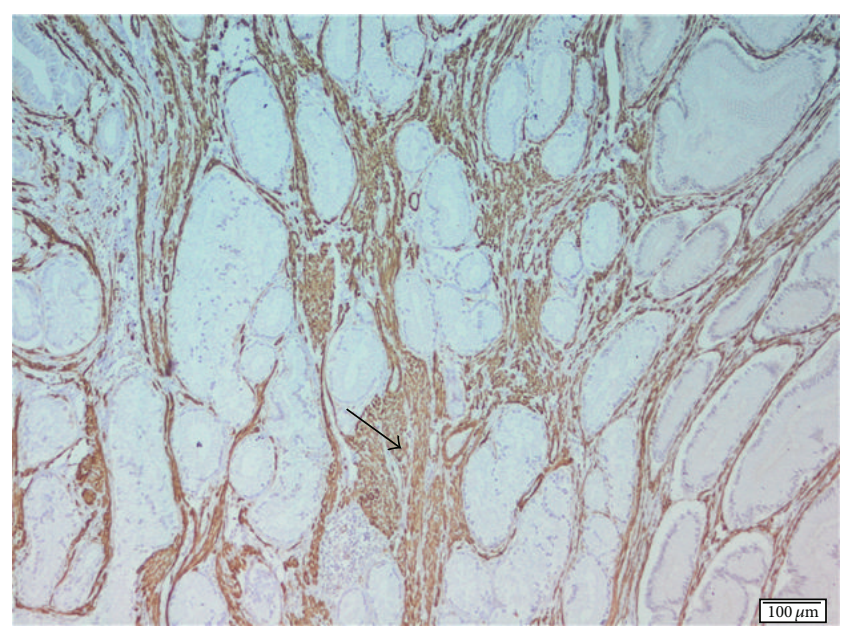

FIGURE 4: Immunohistochemistry showing hyperplastic cells with diffuse and strong positivity with smooth muscle actin (SMA) (arrow), while the gastric glands epithelium was negative. Immunoperoxidase-diaminobenzidine stain, Mayer hematoxylin counterstain. Bar $=100 \mu \mathrm{m}$.

To the best of our knowledge, only 28 cases of human gastric solitary hamartomatous polyps were so far documented in the literature [15]. In animals, gastric smooth muscle hamartoma has only been described in the abomasum of a calf [5] and adjacent to the lower gastroesophageal sphincter in a cat [4]. Our current case demonstrated extensive branching of the alpha actin stained smooth muscle fibers from muscularis mucosae to the mucosal layer between gastric glands and foveolae and their atrophy. These characteristics thus need to be differentiated from hyperplastic and inflammatory types. This constitutes the first report of this kind of lesion in canine stomach. In the majority of cases, these lesions are probably subdiagnosed and classified as hyperplastic gastric polyps because endoscopic biopsies are superficial and fail to obtain the representative features of the lesion or because immunohistochemistry studies are not performed.

On the other hand, hamartomatous polyposis syndromes are a diverse group of inherited conditions grouped together because they exhibit hamartomatous rather than epithelial polyp histology. If in human pathology several reports revealed the malignant potential of the hamrtoamatous gastric polyps associated with Peutz-Jeghers syndrome, Cowden syndrome, juvenile polyposis, and PTEN hamartoma tumour syndrome [16], in dogs, a malignant transformation was found only in the hyperplastic polyps [9]. The progression of these polyps to cancer is not well understood, but a possible malignant transformation of the epithelial components was debated [17]. No changes of the epithelial cells of both foveole and glands were observed in the present case.

Brown et al. (1994) described the presence of hamartomatous polyps in the intestine of two dogs, reporting for the first time the occurrence of such lesions in domestic animals [18]. A case of colorectal hamartomatous polyposis and ganglioneuromatosis with PTEN duplication affecting a Great Dane puppy was recently documented. In this case, similar to what occurs in human Cowden syndrome, a possible mutation was investigated in the PTEN gene; however, such mutation was not proven [19].

In human medicine, a gastric hamartomatous polyp is a well-defined entity, already described and recognized by WHO. A more precise and elaborated gastric classification scheme is needed in veterinary medicine, where the histological and immunophenotypic features of a lesion should be taken together in consideration. Thus, being the third case described affecting different animal species, this entity should be included in this classification. On the other hand, an important challenge will be to determine the mechanisms involved for development of the hamartomatous gastric polyps in dogs and their correlation with a possible genetic mutation.

To our knowledge, this is the first report of smooth muscle hamartomas mimicking multiple gastric polyps in dogs. If the smooth muscle hamartomatous gastric polyps could represent a predisposing factor to the development of malignancy in dogs, a generic hypothesis remains which needs further studies for confirmation.

\section{Authors' Contribution}

Taulescu Marian and Irina Amorim contributed equally to this work.

\section{Acknowledgments}

I. Amorim (SFRH/BD/76237/2011) acknowledges FCT, the Portuguese Foundation for Science and Technology, for financial support. The Institute of Molecular Pathology and Immunology of the University of Porto (IPATIMUP) is an Associate Laboratory of the Portuguese Ministry of Science, Technology and Higher Education and is partially supported by FCT. 


\section{References}

[1] K. W. Head, J. M. Cullen, R. R. Dubielzig et al., "Histological histological classification of tumors of the alimentary system of domestic animals," in International Histological Classification of Tumors of Domestic Animals, F. Y. Schulman, Ed., pp. 75-111, AFIP, Washington, DC, USA, 2003.

[2] A. Diana, D. G. Penninck, and J. H. Keating, "Ultrasonographic appearance of canine gastric polyps," Veterinary Radiology and Ultrasound, vol. 50, no. 2, pp. 201-204, 2009.

[3] S. Kuan, K. Hoffmann, and P. Tisdall, "Ultrasonographic and surgical findings of a gastric hyperplastic polyp resulting in pyloric obstruction in an 11-week-old French Bulldog," Australian Veterinary Journal, vol. 87, no. 6, pp. 253-255, 2009.

[4] T. J. Smith, W. I. Baltzer, C. G. Ruaux, J. R. Heidel, and P. Carney, "Gastric smooth muscle hamartoma in a cat," Journal of Feline Medicine and Surgery, vol. 12, no. 4, pp. 334-337, 2010.

[5] M. Yamaguchi, N. Machida, K. Mitsumori, M. Nishimura, and Y. Ito, "Smooth muscle hamartoma of the abomasum in a calf," Journal of Comparative Pathology, vol. 130, no. 1, pp. 66-69, 2004.

[6] C. C. Morse and D. W. Richardson, "Gastric hyperplastic polyp in a horse," Journal of Comparative Pathology, vol. 99, no. 3, pp. 337-342, 1988.

[7] R. P. Happe, I. Van Der Gaag, W. C. Wolvekamp Th., and J. Van Toorenburg, "Multiple polyps of the gastric mucosa in two dogs," Journal of Small Animal Practice, vol. 18, no. 3, pp. 179189, 1977.

[8] Y. P. Do and G. Y. Lauwers, "Gastric polyps: classification and management," Archives of Pathology and Laboratory Medicine, vol. 132, no. 4, pp. 633-640, 2008.

[9] M. Gualtieri, M. G. Monzeglio, E. Scanziani, and C. Domeneghini, "Pyloric hyperplastic polyps in the French Bulldog," European Journal of Companion animal Practice, vol. 6, pp. 51-57, 1996.

[10] C. C. Brown, D. C. Baker, and I. K. Barker, "Alimentary system," in Pathology of Domestic Animals, K. V. F. Jubb, P. C. Kennedy, and N. Palmer, Eds., pp. 1-296, Saunders Elsevier, Philadelphia, $\mathrm{Pa}, \mathrm{USA}, 2007$.

[11] Y. Kim, S. Reinecke, and D. E. Malarkey, "Cutaneous angiomatosis in a young dog," Veterinary Pathology, vol. 42, no. 3, pp. 378-381, 2005.

[12] M. Sakurai, T. Morita, H. Kondo, T. Uemura, A. Haruna, and A. Shimada, "Cerebral vascular hamartoma with thrombosis in a dog," Journal of Veterinary Medical Science, vol. 73, no. 10, pp. 1367-1369, 2011.

[13] G. Chanoit, K. G. Mathews, B. W. Keene, M. T. Small, and K. Linder, "Surgical treatment of a pulmonary artery vascular hamartoma in a dog," Journal of the American Veterinary Medical Association, vol. 240, no. 7, pp. 858-862, 2012.

[14] K. M. Zbuk and C. Eng, "Hamartomatous polyposis syndromes," Nature Clinical Practice Gastroenterology and Hepatology, vol. 4, no. 9, pp. 492-502, 2007.

[15] J. S. Jin, J. K. Yu, T. Y. Tsao, and L. F. Lin, "Solitary gastric PeutzJeghers type stomach polyp mimicking a malignant gastric tumor," World Journal of Gastroenterology, vol. 18, pp. 18451848, 2012.

[16] A. Gammon, K. Jasperson, W. Kohlmann, and R. W. Burt, "Hamartomatous polyposis syndromes," Best Practice and Research, vol. 23, no. 2, pp. 219-231, 2009.

[17] J. R. Jass, "Pathology of polyposis syndromes with special reference to juvenile polyposis," in Hereditary Colorectal Cancer,
J. Utsunomiya and H. T. Lynch, Eds., p. 343, Springer, Tokyo, Japan, 1990.

[18] P. J. Brown, S. M. Adam, P. R. Wotton, C. Gibbs, and R. H. Swan, "Hamartomatous polyps in the intestine of two dogs," Journal of Comparative Pathology, vol. 110, no. 1, pp. 97-102, 1994.

[19] I. Bemelmans, S. Küry, O. Albaric et al., "Colorectal hamartomatous polyposis and ganglioneuromatosis in a dog," Veterinary Pathology, vol. 48, no. 5, pp. 1012-1015, 2011. 

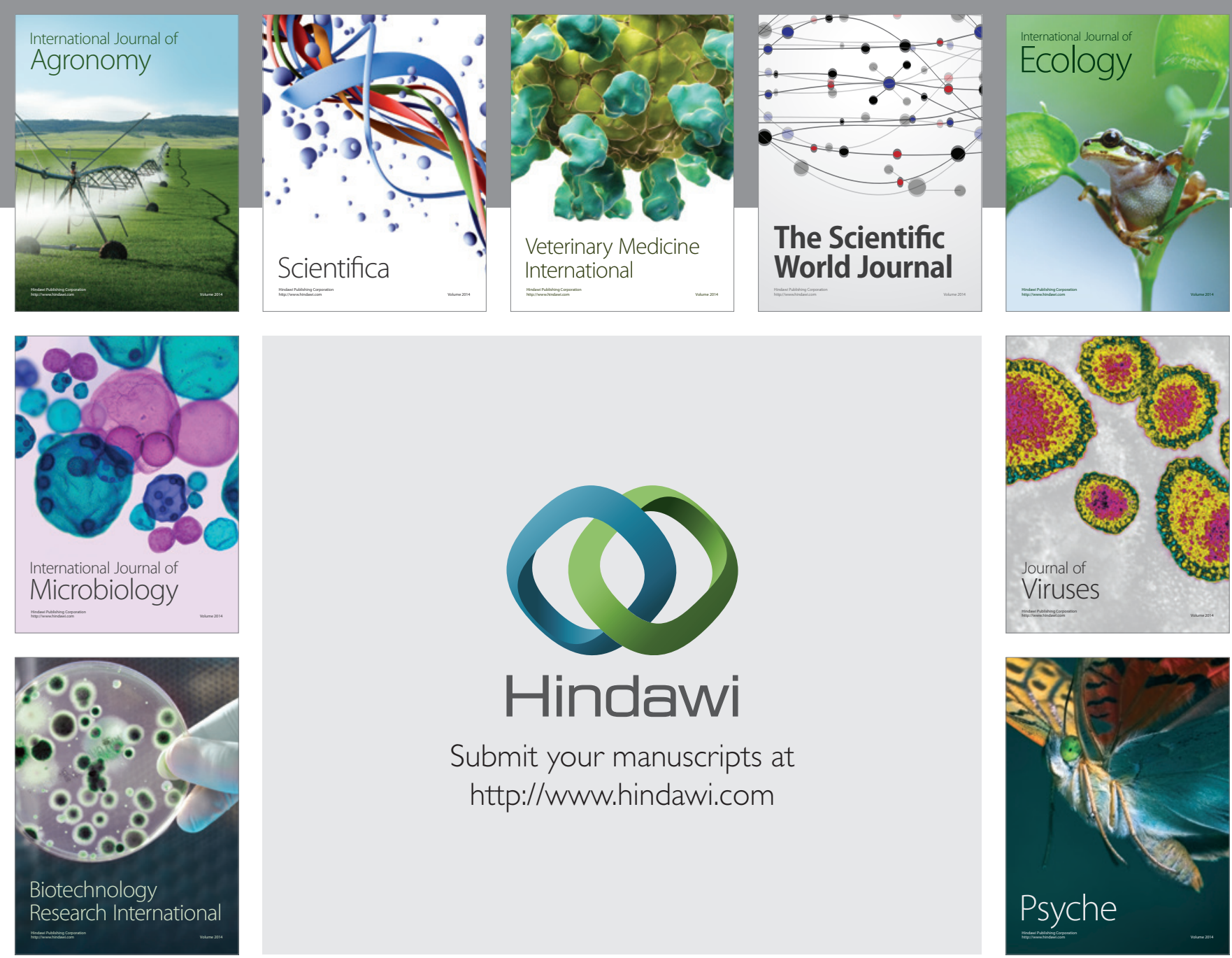

Submit your manuscripts at http://www.hindawi.com
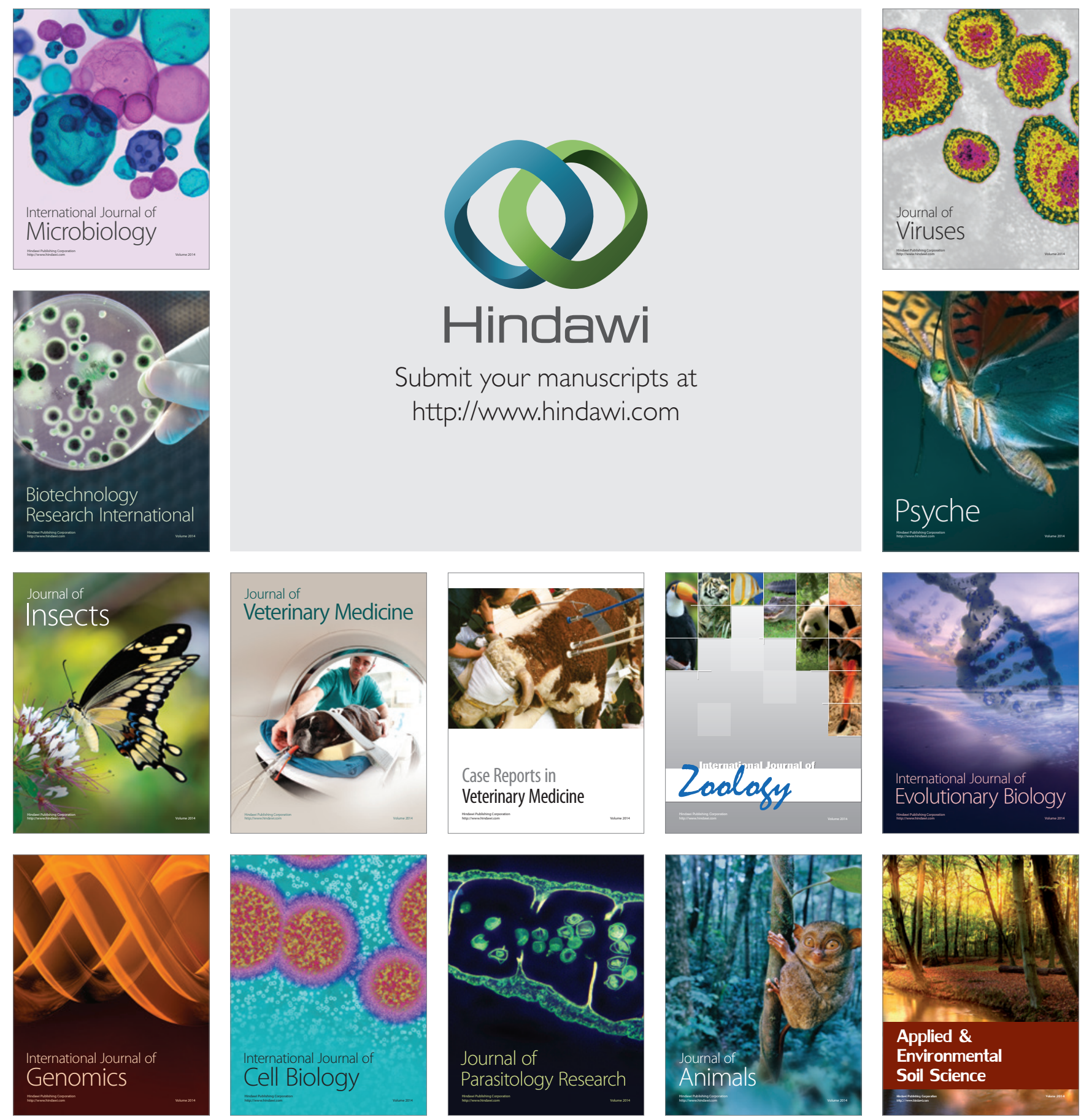